\title{
A single minute lesion around the ventral respiratory group in medulla produces fatal apnea in cats
}

\author{
J.H. Hsieh a,b, Y.C. Chang ${ }^{\text {b }}$, C.K. Su ${ }^{\text {a }}$, J.C. Hwang ${ }^{\text {c }}$, C.T. Yen ${ }^{\text {d }}$, C.Y. Chai a,* \\ ${ }^{a}$ Institute of Biomedical Sciences, Academia Sinica, Taipei, 11529, Taiwan \\ ${ }^{\mathrm{b}}$ Institute of Biomedical Engineering, Chung Yuan Christian University, Taiwan \\ ${ }^{\mathrm{c}}$ Department of Biology, National Taiwan Normal University, Taiwan \\ d Department of Zoology, National Taiwan University, Taiwan
}

Received 10 November 1997; revised 24 April 1998; accepted 2 June 1998

\begin{abstract}
In 35 adult cats anesthetized with intraperitoneal chloralose and urethane, the ventrolateral medulla was explored by microinjection of kainic acid (KA, $24 \mathrm{mM}, 200 \mathrm{nl}$ ) with metal electrode-tubing or glass micropipette to determine regions which elicit persistent apnea. Persistent apnea is defined as: (1) In spontaneously breathing cats, termination of respiration over 3 min with a decrease of the mean systemic arterial pressure (MSAP) to $25 \mathrm{~mm} \mathrm{Hg}$. (2) In animals under artificial ventilation and paralyzed by gallamine, cessation of bilateral phrenic nerve (PNA) activities over $25 \mathrm{~min}$. The apnea producing area was located dorsal to the rostral pole of the lateral reticular nucleus, ventromedial to the ambiguous nucleus and immediately caudal to the retrofacial nucleus. Functionally, this region includes the rostral part of the ventral respiratory group (rVRG) encompassing the pre-Bötzinger area. We define this region as the VRG apnea producing area (VRG-Apa). Fatal apneusis was observed under following conditions: (1) Persistent apnea was produced after a single KA microinjection in one side of the VRG-Apa (5 animals). Microinjection of sodium glutamate (0.25 M, 70-200 $\mathrm{nl})$ in the same area produced only brief apnea, while microinjection of kynurenic acid (0.1 M, $200 \mathrm{nl})$ showed little effect on the respiration but slightly increased the SAP. (2) Positioning an electrode nearby but not in the VRG-Apa with or without KA injection did not produce apnea. But when a second electrode insertion to the opposite VRG-Apa immediately produced persistent apnea even without KA injection (6 animals). (3) Midsagittal division of the medulla $0-5 \mathrm{~mm}$ rostral to the obex produced persistent silence of PNA on both sides in artificial ventilated animals ( 7 animals), while similar division $0-5 \mathrm{~mm}$ caudal to the obex (4 animals) produced a brief but reversible quiescence of PNA. In conclusion, findings of the present study support the existence of a restricted region of VRG-Apa. VRG-Apa on both sides are closely connected, and integrity of both VRG-Apa is essential for normal respiration. (C) 1998 Elsevier Science B.V. All rights reserved.
\end{abstract}

Keywords: Ventral respiratory group; Apnea; Phrenic nerve activity; Midsagittal division in medulla

\section{Introduction}

The importance of the lower brainstem in respiratory rhythmogenesis has been the subject of interest over a century (Wang, 1980), and the region around the ventral respiratory group (VRG) of the medulla has been found to be the site most important for such action (Adams et al., 1988; Feldman and Smith, 1989; Onimaru et al., 1989; Onimaru, 1995; Paton et al., 1994). We have observed that (Chai et al., 1965) in spontaneously breathing cats under urethane anesthesia, local heating the lateral reticular for-

\footnotetext{
* Corresponding author. Tel.: +886 2 27899105; fax: +8862 27829224.
}

mation of the medulla close to the VRG with high frequency current $(3700 \mathrm{KC})$ produced a temperature-dependent but reversible decrease in respiratory tidal volume close to apnea. Heating the anterior hypothalamus (a structure essential for body temperature integration) with the same current increased respiratory rate without decreasing tidal volume. Microinjection of sodium glutamate (Glu) into the medulla, particularly the rostral ventrolateral region, produced a marked inhibition of the phrenic nerve activity (PNA) lasting for $500 \mathrm{~s}$ (Hwang et al., 1992; Su et al., 1992).

In our experiments dating back to the early 1960's, fatal-respiratory failure often occurred consequent to electrode insertion in the medulla, particularly in its ventral 
region (unpublished data). In current preliminary search (Hwang, 1994) using 11 spontaneously breathing cats, we observed that in 8 of these animals, insertion of a metal electrode-tubing close to, or slightly beneath the ambiguous nucleus at the level caudal to the facial nucleus, easily induced respiratory irregularity and intermittent apnea. In 4 of these 8 animals, unilateral microinjection of kainic acid (KA, $24 \mathrm{mM}, 200 \mathrm{nl}$ ) into this area produced apnea, concomitant with hypertension, then gradual falling of SAP, and eventually death of the animals. In contrast, in the remaining 3 cats, microinjecting the same amount of KA into the region around the solitary nucleus did not cause death of the animals despite the occurrence of intermittent apnea accompanied with cardiac irregularity.

In the present study, we focused our attention on localizing precisely the area of VRG wherein persistent apnea could be induced by penetration with conventional metal electrode or by KA microinjection. We refer to this VRG that can produce apnea as VRG-apnea producing area (VRG-Apa). Persistent apnea is defined in terms of the following conditions. Under spontaneous breathing, respiration stopped over $5 \mathrm{~min}$ with a drop of the mean SAP (MSAP) to or below $25 \mathrm{~mm} \mathrm{Hg}$, or under artificial ventilation with neuromuscular blocking agent (gallamine) the

\section{YC970203}

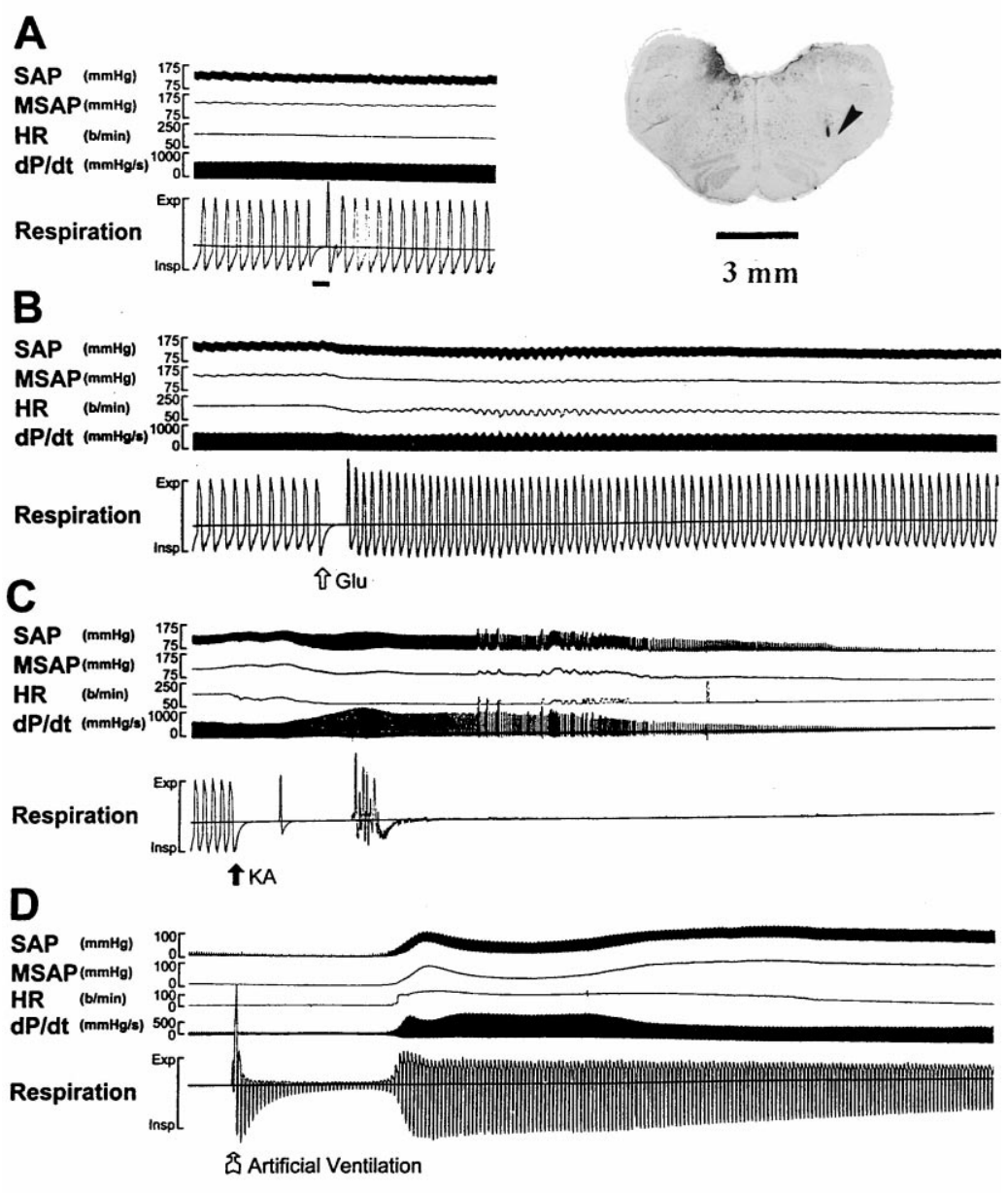

\footnotetext{
$\overline{45 \mathrm{sec}}$

Fig. 1. Microinjection of KA in VRG-Apa produced apnea. In a cat (970203): (A) Electrical stimulation (50 $\mu$ A) on VRG-Apa with a metal electrode-tubing briefly inhibited the respiration. (B) Microinjection of a low dose of Glu (100 nl) into the same point initially produced transient inhibition, followed by prolonged enhancement of respiration associated with slight decreases in SAP and HR. (C) Chemical lesion of neurons in the same VRG-Apa by microinjection of KA ( $200 \mathrm{nl})$ immediately abolished the spontaneous breathing accompanied at first with a very slight increase in SAP of short duration then followed by a progressive decrease in the resting SAP and HR. (D) Fifteen min after this procedure and the animal was rescued by artificial ventilation the resting SAP and HR returned gradually to its control level. However, the animal could not regain breathing despite artificial ventilation for $3 \mathrm{~h}$. In this and the following figures only photo pictures of unstained brain slices are presented. Some chemical crystals (dots in the picture) were left in the tissue. Abbreviations: VRG-Apa, ventral respiratory group-Apnea producing area; Glu, glutamate; KA, kainic acid; SAP, systemic arterial blood pressure; MSAP, mean SAP; dp/dt, indirect cardiac contractile force; HR, heart rate; Resp, respiration; Exp, expiration; Insp, inspiration.
} 
phrenic nerve activity (PNA) on both sides ceased over 25 min.

\section{Methods and materials}

\subsection{General procedure}

Experiments were performed on adult cats of either sex weighing between 2.2 and $2.6 \mathrm{~kg}$, anesthetized intraperitoneally with a mixture of chloralose $(40 \mathrm{mg} / \mathrm{kg})$ and urethane $(400 \mathrm{mg} / \mathrm{kg})$. The general experimental procedures have been described previously (Chai et al., 1988). These included cannulation of the femoral artery for monitoring the SAP and HR, cannulation of the right femoral vein for drug administrations, and tracheal intubation for monitoring changes of the end-expiratory $\mathrm{CO}_{2}$ concentration (Capnograph, Gould, Cleveland, $\mathrm{OH}$ ) to record the respiratory movement during spontaneous breathing. The end-expiratory $\mathrm{CO}_{2}$ concentration was maintained at 3.5$4.0 \%$ in paralyzed animals (gallamine triethiodide, 5 $\mathrm{mg} / \mathrm{kg}$ per hour, i.v.) receiving artificial ventilation during phrenic nerve recording. The rectal temperature of the animal was maintained at $37.5^{\circ} \mathrm{C}$ by a thermo-controlled heating pad (Harvard, Millis, MA). All parameters were recorded on a polygraph (model ES 1000, Gould).

\subsection{Neural recording}

Phrenic nerves on both sides were exposed, dissected free from the surrounding connective tissues, cut distally, placed on a bipolar platinum electrode and immersed in mineral oil.

Phrenic nerve activities (PNA) were monitored following the procedures as described previously (Hwang et al., 1992; Wang et al., 1996). PNA were amplified, filtered $(0.1-3 \mathrm{Kz}$ ), and stored on a tape recorder (model DR-886, Neuro Data, New York, NY) for later analysis. During

YC970315

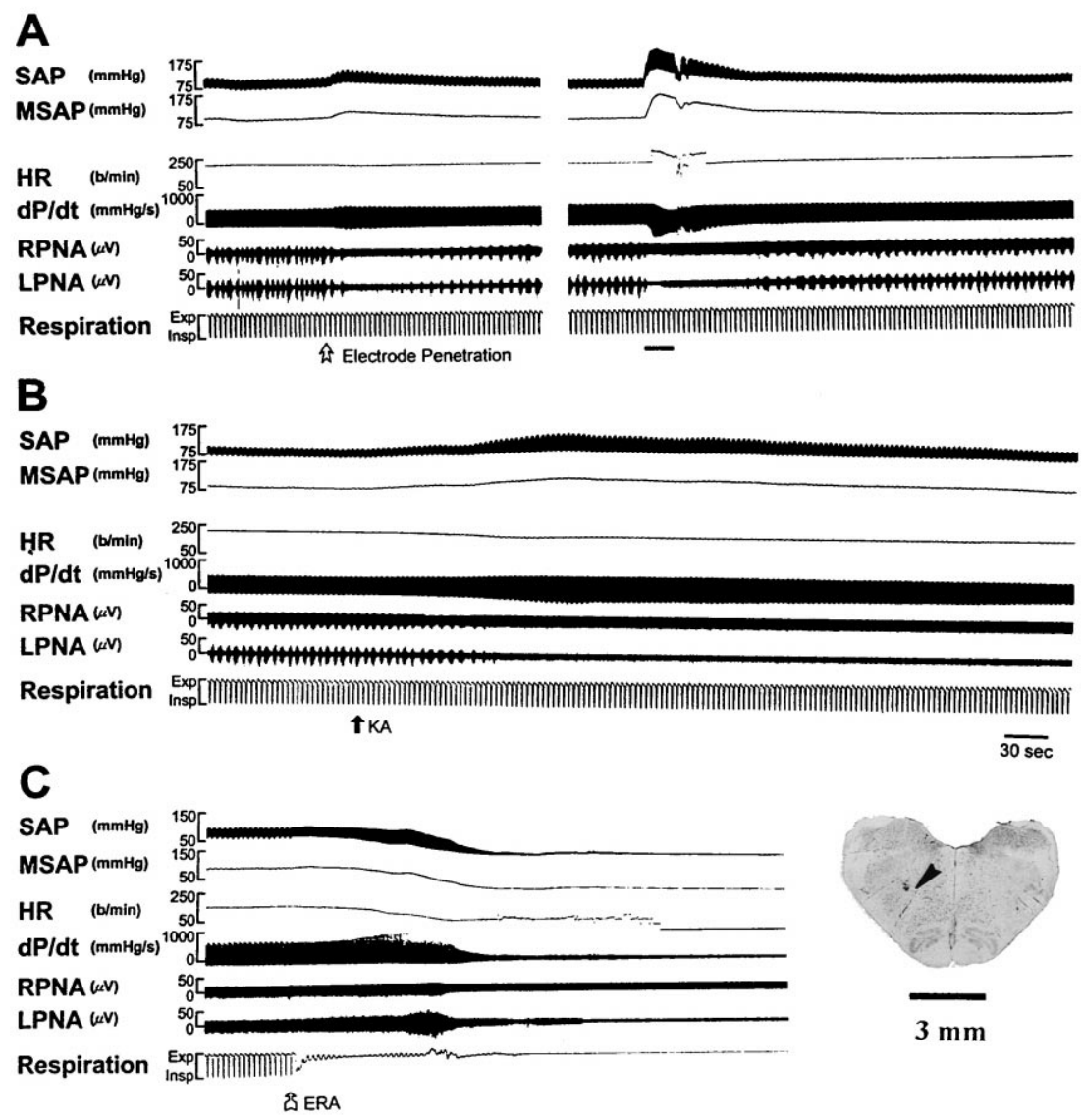

Fig. 2. KA abolishes the phrenic nerve activity (PNA) when it was microinjected into the VRG-Apa. In a cat (970315) under anesthesia and artificial ventilation. (A) The animal was under gallamine. Penetration of the electrode into the VGR-Apa alone produced a brief inhibition of the PNA on both sides and a slight increase in SAP. Electrical stimulation $(50 \mu \mathrm{A})$ of the same VRG-Apa produced similar inhibition of the PNA but greater increases of SAP and HR. (B) Microinjection of KA (200 nl) into the same VRG-Apa produced a complete inhibition of the PNA concomitant with moderate but slow rise in SAP. Gallamine was discontinued shortly after the silence of PNA. (C) The PNA did not re-appear after KA despite artificial ventilation for $3 \mathrm{~h}$. Discontinuation of artificial ventilation and exposed the animal to room air (ERA) produced progressive falling of SAP to zero. The animal did not show apparent sign of struggle because of anoxia. Abbreviations: RPNA, right phrenic nerve activity; LPNA, left phrenic nerve activity. 
recording, animals were under artificial ventilation (Harvard ventilator) and paralyzed with gallamine trithiodide $(5 \mathrm{mg} / \mathrm{kg}$ per hour).

\subsection{Brain stimulations and lesioning}

Animals were placed in a prone position and the head of the animal was fixed in a David-Kopf stereotaxic instrument. After craniotomy, the brain was exposed for stimulation through a dorsal to ventral approach. Two types of electrodes were used. One is metal electrode prepared by gluing together a pair of electrode tubings with outside diameter $0.27 \mathrm{~mm}$. Each tubing, insulated except for $200 \mu \mathrm{m}$ at the tip, was connected through a polyethylene tube (PE 10) to a Hamilton syringe filled with different chemicals, i.e. Glu $(0.25 \mathrm{M})$; kainic acid (KA, $24 \mathrm{mM})$; kynurenic acid (KYN, $0.1 \mathrm{M})$. Under this arrangement, reactive points in the medulla could be explored monopolarly by electrical stimulation and by injection of Glu (30-100 nl) to confirm the existence of cell bodies. Injection of KA (200 $\mathrm{nl})$ was used to produce lesion at the same point (Chai et al., 1988). All reagents were dissolved in artificial cerebrospinal fluid (in mM, 124 $\mathrm{NaCl}, 2 \mathrm{KCl}, 2 \mathrm{MgCl}_{2}, 2 \mathrm{CaCl}_{2}, 1.25 \mathrm{KH}_{2} \mathrm{PO}_{4}, \mathrm{NaHCO}_{3}$, at $\mathrm{pH}$ 7.4) containing $1 \%$ pontamine sky blue. The injection volume was 100-200 nl. Control microinjection in agar gel showed that $30 \mathrm{nl}$ produced a stain in diameter of $0.1 \mathrm{~mm}, 70 \mathrm{nl}$ in $0.15 \mathrm{~mm}, 100 \mathrm{nl}$ in $0.16 \mathrm{~mm}$, while 200 $\mathrm{nl}$ in $0.22 \mathrm{~mm}$.

The alternate electrode was a double-barrel glass micropipette. One barrel (tip diameter 40-50 $\mu \mathrm{m}$ ) for electrical stimulation was filled with $\mathrm{NaCl}(3 \mathrm{M})$ with a platinum wire inserted, while the other was filled with Glu, KA or KYN solution. Each barrel was connected to a pneumatic pump (Pneumatic Pressure System, model PPS-2, Medical Systems, Greenvale, NY, USA) for microinjection in volumes of 50-150 nl.

The electrode or micropipette was positioned perpendicular to the floor of the medulla with an inclination of the electrode carrier at an angle of $34^{\circ}$ toward the stereotaxic

\section{YC970127}

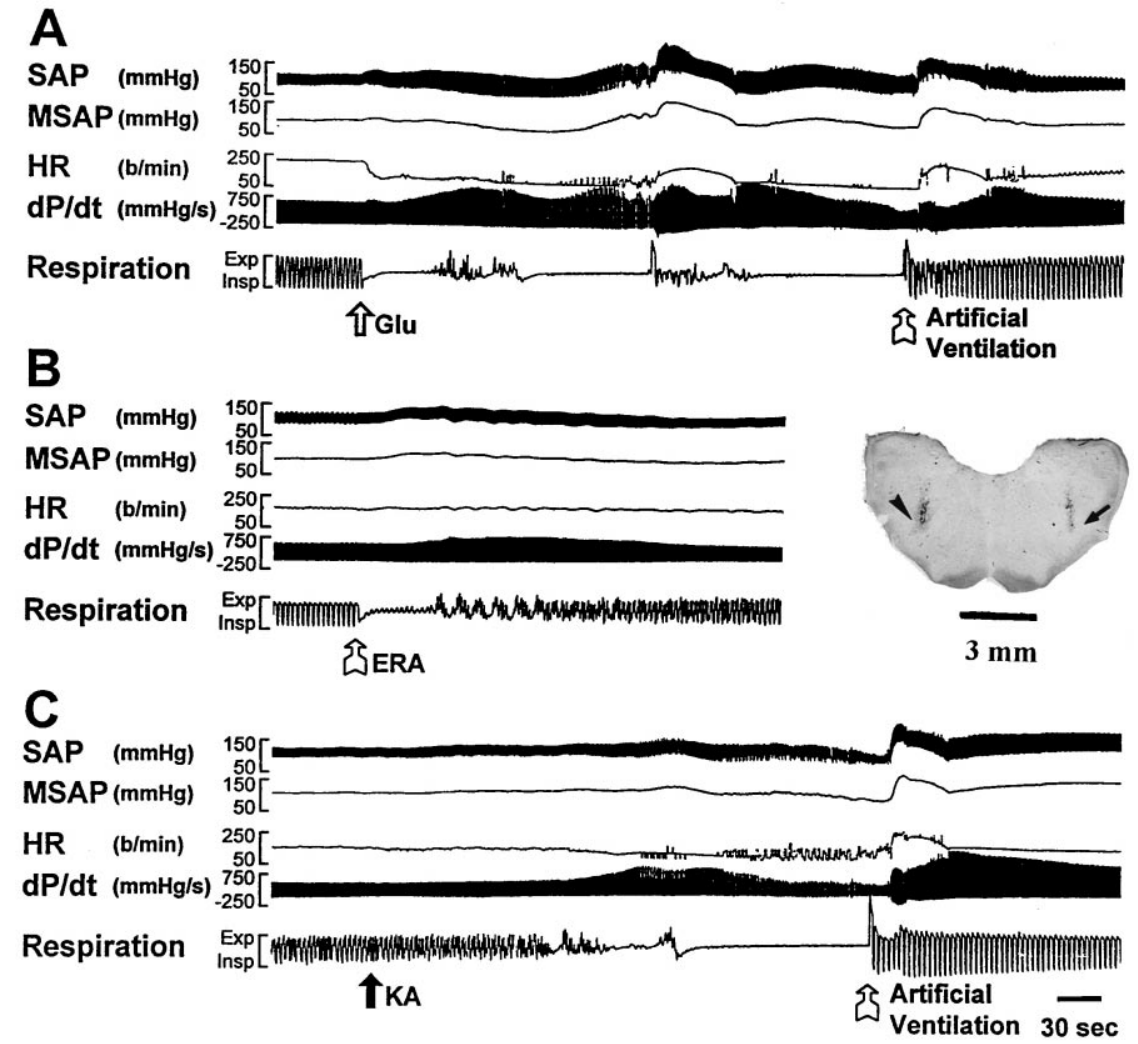

Fig. 3. A high dose of Glu microinjected into the VRG-Apa also produced apnea. In an anesthetized animal breathed spontaneously (970127), two penetrations were made on the medulla by a double barrel micropipette; one (right) lateral to the VRG-Apa which did not produce apparent response to electrical stimulation (not shown), another (left) on the VRG-Apa. (A) Microinjection of a large dose of Glu (200 nl) through a metal electrode-tubing into the VRG-Apa previously identified by electrical stimulation produced immediate apnea intermittent with fast but shallow respiratory movements associated with fluctuation, decrease and increase of SAP and bradycardia. The situation was corrected by placing the animal under artificial ventilation. (B) Ten min after this procedure, the resting SAP returned to its control level. The animal could not breath shortly after discontinuation of artificial ventilation and exposure to room air (ERA) but gradually regained spontaneous breathing. (C) Microinjection of KA (200 nl) into the same VRG-Apa, decreased respiration and eventually apnea accompanied immediately with a progressive decrease of the resting SAP. The animal was put back on artificial ventilation but spontaneous breathing did not return even continuation of such procedure for $2 \mathrm{~h}$. 
frame. Stimulating current was generated by a Grass S48 rectangular pulse stimulator and delivered via a constantcurrent unit. The parameters were $80 \mathrm{~Hz}, 0.5 \mathrm{~ms}, 50-100$ $\mu \mathrm{A}$ for a $15 \mathrm{~s}$ period.

\subsection{Midsagittal division of the medulla}

In 7 cats, the medulla was midsagitally divided into two halves from the midline using a knife, made from a razor blade, at the level of $0-5 \mathrm{~mm}$ rostral to the obex. In other 4 cats, midsagittal division was made $0-5 \mathrm{~mm}$ caudal to the obex.

\subsection{Histological confirmation}

At the end of each experiment, the brain was removed and immersed in $10 \%$ formalin solution for 2-3 days. Frontal sections of the lower brainstem $(50 \mu \mathrm{M}$ in thickness) were cut using a cryostat (Reichert-Jung 2800, Frigocut E). Two sets of brain slices were prepared. One set was unstained, without cover slide, for better demonstration of the needle track and the blue stain of pontamine sky blue subsequent to microinjection. The other sections was stained with cresyl violet for histological detail. Only unstained-brain slices are shown in the accompanying figures.

\section{Results}

\subsection{The VRG-Apa}

\subsubsection{Localization of the VRG-Apa}

Precise localization of the VRG-Apa was explored first by electrical stimulation, followed by chemical stimulations. Such experiments were carried out in 19 cats under spontaneous respiration and 16 cats under artificial ventilation. The stereotaxic coordinates were $2.0-3.2 \mathrm{~mm}$ rostral to the obex, $3.0-3.2 \mathrm{~mm}$ lateral from the midline, and within a depth of $3.5-4.5 \mathrm{~mm}$ from the dorsal surface of the medulla. Histological examination of the frontal sections revealed that 35 VRG-Apa points were located dorsal

\section{YC970214}

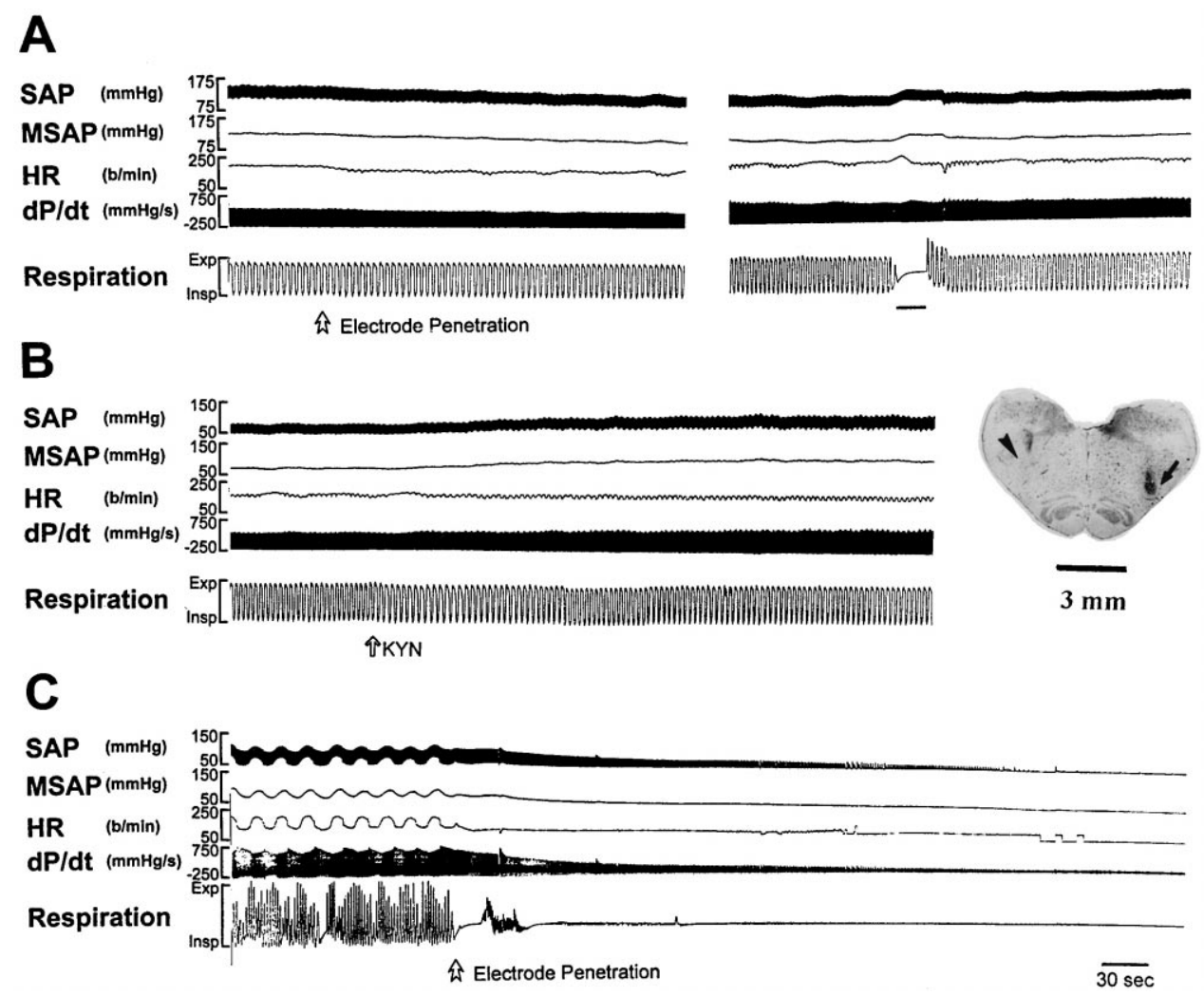

Fig. 4. KYN to VRG-Apa did not induce apnea. In an anesthetized but spontaneous breathing cat (970214): (A) Penetration of a metal electrode-tubing on VRG-Apa slightly decreased the SAP (Left panel). Electrical stimulation $(50 \mu \mathrm{A})$ of the same point in VRG-Apa produced brief inhibition of respiration and slight increase in SAP and HR (Right panel). (B) Microinjection KYN (200 nl) into the same point did not produce apparent changes in respiration. (C) This point was further lesioned by direct current $(1 \mathrm{~mA})$ for $40 \mathrm{~s}$. Note the resulting saw like SAP and fluctuation of the respiration as shown in the beginning of the trace in $\mathrm{C}$. The animal was left undisturbed for $10 \mathrm{~min}$, and another electrode penetration was made in the opposite VRG-Apa. The second penetration alone produced an immediate apnea accompanied with progressive decline in SAP and HR. During the apnea period no sign of struggling was observed even when the SAP and HR eventually reached zero. In this and the following figures: KYN, kynurenic acid. 
to the rostral pole of the lateral reticular nuclei (LRN), ventromedial to the ambiguous nucleus, and immediately caudal to the retrofacial nucleus. Among these 21 points (located at the level showing apparent cluster of LRN) were distributed slightly caudal to the remaining 14 points. Functionally, these VRG-Apa points include the rostral part of rostral VRG (rVRG) $(21 / 35)$ and encompass the pre-Bötzinger area $(14 / 35)$.

\subsubsection{Electrical stimulation of the VRG-Apa}

In spontaneously breathing cats, electrical stimulation $(50 \mu \mathrm{A})$ of the VRG-Apa immediately caused brief apnea (Fig. 1A, Fig. 4A), but respiration resumed immediately after the stimulation was terminated. Similar inhibitory effects on PNA were observed in cats under artificial ventilation. In artificially ventilated cats with phrenic nerve recording, electrical stimulation of the VRG-Apa completely inhibited bilateral PNA (Fig. 2A, left panel, inhibition lasting for $72 \mathrm{~s}$ ). In either group, stimulation produced only slight increase in SAP.

\subsubsection{Glu stimulation of the VRG-Apa}

In spontaneously breathing cats, microinjection of low dose Glu (100 nl) into the VRG-Apa produced only tran- sient apnea, and then a period of tachypnea as shown in Fig. 1B. Changes of SAP and HR in these experiments were slight. In contrast to the effect of Glu in low dose, delivery of a higher dose of Glu $(200 \mathrm{nl})$ into the VRG-Apa increased the SAP from $88 \pm 23$ to $119 \pm 32 \mathrm{~mm} \mathrm{Hg}, \mathrm{HR}$ decreased from $221 \pm 10$ to $120 \pm 60 \mathrm{bpm}$, while respiration became irregular and intermingled with apnea and fast shallow pattern (Fig. 3A). These animals resumed spontaneous breathing but with an irregular pattern after a short period of artificial ventilation (Fig. 3B). Further damage of this region by KA might produce persistent apnea and death of the animal (Fig. 3C).

\subsubsection{KA lesioning the VRG-Apa}

Three types of change in SAP following microinjection of KA into the VRG-Apa were observed during spontaneous respiration. Type 1: KA injection into the VRG-Apa produced an initial but brief increase of SAP, then followed by apnea. In 3 cats, after identification of the VRG-Apa by electrical stimulation $(50 \mu \mathrm{A})$ and/or a low dose of Glu (100 nl), KA was then microinjected into the same VRG-Apa. On average, $121 \pm 67$ s after KA, MSAP increased from $92 \pm 12$ to $127 \pm 14 \mathrm{~mm} \mathrm{Hg}$, HR de-

\section{YC970217}

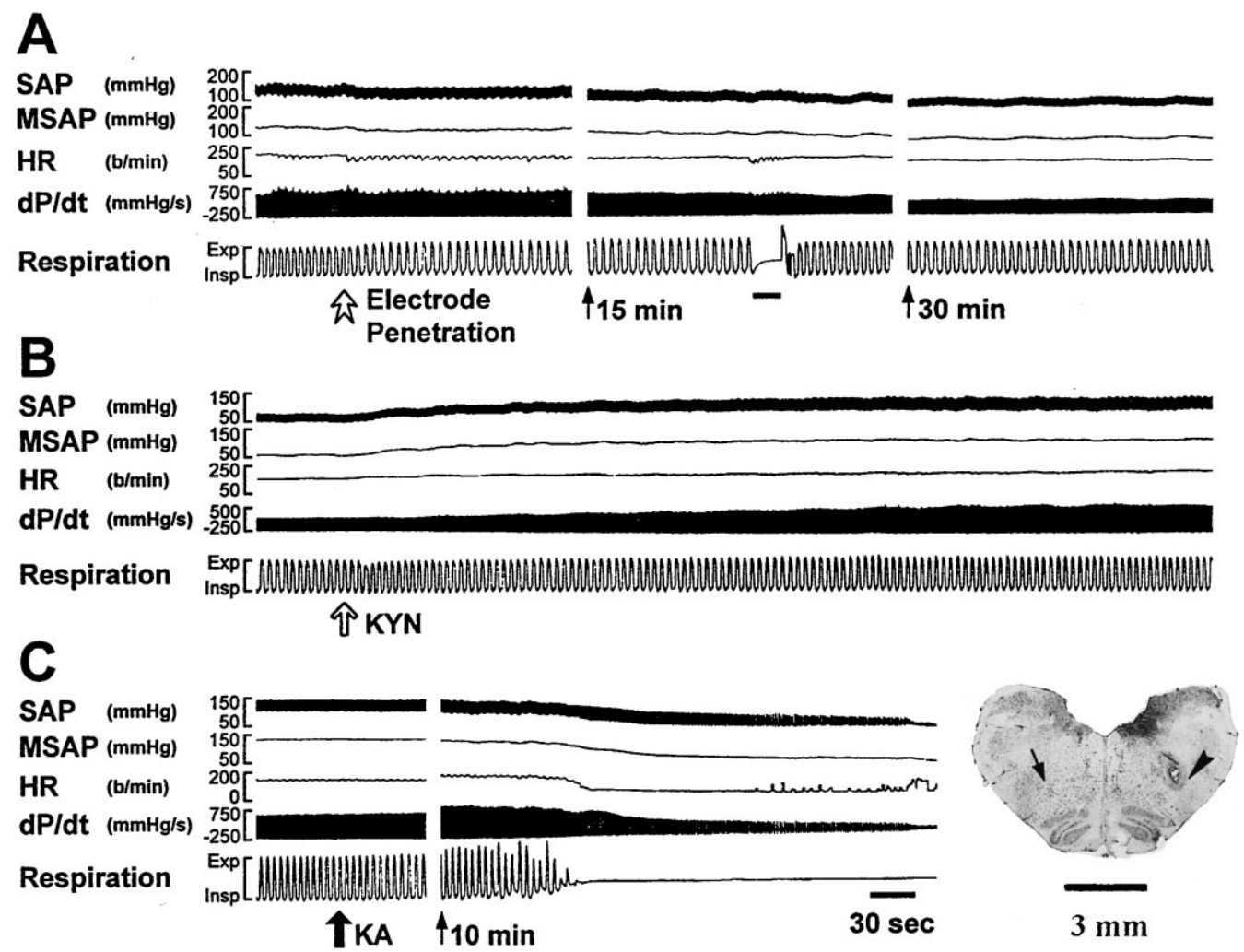

Fig. 5. A second microinjection of KA at a point in VRG-Apa contralateral to a point in ventrolateral medulla previously receiving KYN microinjection caused persistent apnea and death of the animal. In an anesthetized but spontaneous breathing cat (970217): (A) Penetration to the VRG-Apa by a metal electrode-tubing slightly decreased the SAP. Electrical stimulation (50 $\mu \mathrm{A})$ at this point produced a brief inhibition of the respiration and slight decreases in both SAP and HR. (B) Microinjection of KYN (200 nl) into the same point progressively increased the SAP to its original level. (C) Microinjection of KA (200 nl) into the contralateral VRG-APA produced a gradual decline in SAP and HR, and eventually to zero. During the course of apnea, the animal showed no signs of gasping and struggling. 
creased from $130 \pm 25$ to $109 \pm 50 \mathrm{bpm}$ and respiration gradually diminished reaching a state close to apnea (Fig. $3 C)$. If artificial ventilation was not initiated, in an average of $294 \pm 63$ s complete apnea subsequently occurred and the resting SAP dropped to zero. Type 2: In 4 cats, KA injection initially produced little change in SAP or only a slight increase in SAP, then the SAP slowly but progressively decreased (Fig. 1C). Apnea occurred preceding the change of SAP and eventually leading to death of the animal if ventilation was not applied. Type 3: Both respiration and SAP had no significant change within $10 \mathrm{~min}$ $(n=3)$ (Fig. 5C). However, in about $20 \mathrm{~min}$ after KA injection respiration decreased rapidly and finally ceased completely, and in another 6 min later, SAP and HR fell progressively to zero. Histological verification demonstrated that KA injection hit the core of VGR-Apa in experiments of type 1 and 2 while in type 3 the brain lesion was slightly away from the core.

The effect of KA microinjection into the VRG-Apa upon respiration was further examined in another 5 cats. Under artificial ventilation and paralyzed by gallamine, PNA on both sides were recorded. Electrode penetration and electrical stimulation to the VRG-Apa produced brief inhibition of both PNA, associated with slight, moderate, to marked increase in SAP and HR dependent on the extent of involvement of the nearby pressor ventrolateral medulla (Fig. 2A). Microinjection of KA (200 nl) into the same unilateral VRG-Apa produced a complete quiescence of PNA on both sides, and an increase in SAP though accompanied with no or slight bradycardia (Fig. 2B). In these animals gallamine was then discontinued and the animals allowed to recover from the paralysis while continuously under artificial respiration for 3-4 h, yet, the PNA did not return (Fig. 2C).

During the prolonged apnea period after KA lesioning or the mechanical electrode penetration, no sign of struggling (increased muscular tone, body movement, frequent gasping etc.) was observed in either the anesthetized but spontaneously breathing cats, or in the anesthetized but artificially ventilated (after discontinuation of gallamine) cats.

\subsubsection{Effects of KYN microinjection into the VRG-Apa}

In 5 spontaneously breathing cats, microinjection of KYN (0.1 M, $200 \mathrm{nl})$ into the VRG-Apa did not produce significant changes in respiration either in the rate or amplitude (Fig. 4B, Fig. 5B). However, in 4 of 5 electrode penetrations into the VRG-Apa, a prolonged decrease in the resting MSAP from an average of 124-62 $\mathrm{mm} \mathrm{Hg}$ was produced (decrease 49\%) (Fig. 5A). Microinjection of KYN into the same point restored the SAP 12\% (MSAP from 68 to $111 \mathrm{~mm} \mathrm{Hg}$ ) of its control level (Fig. 4B, Fig.

\section{YC970115}

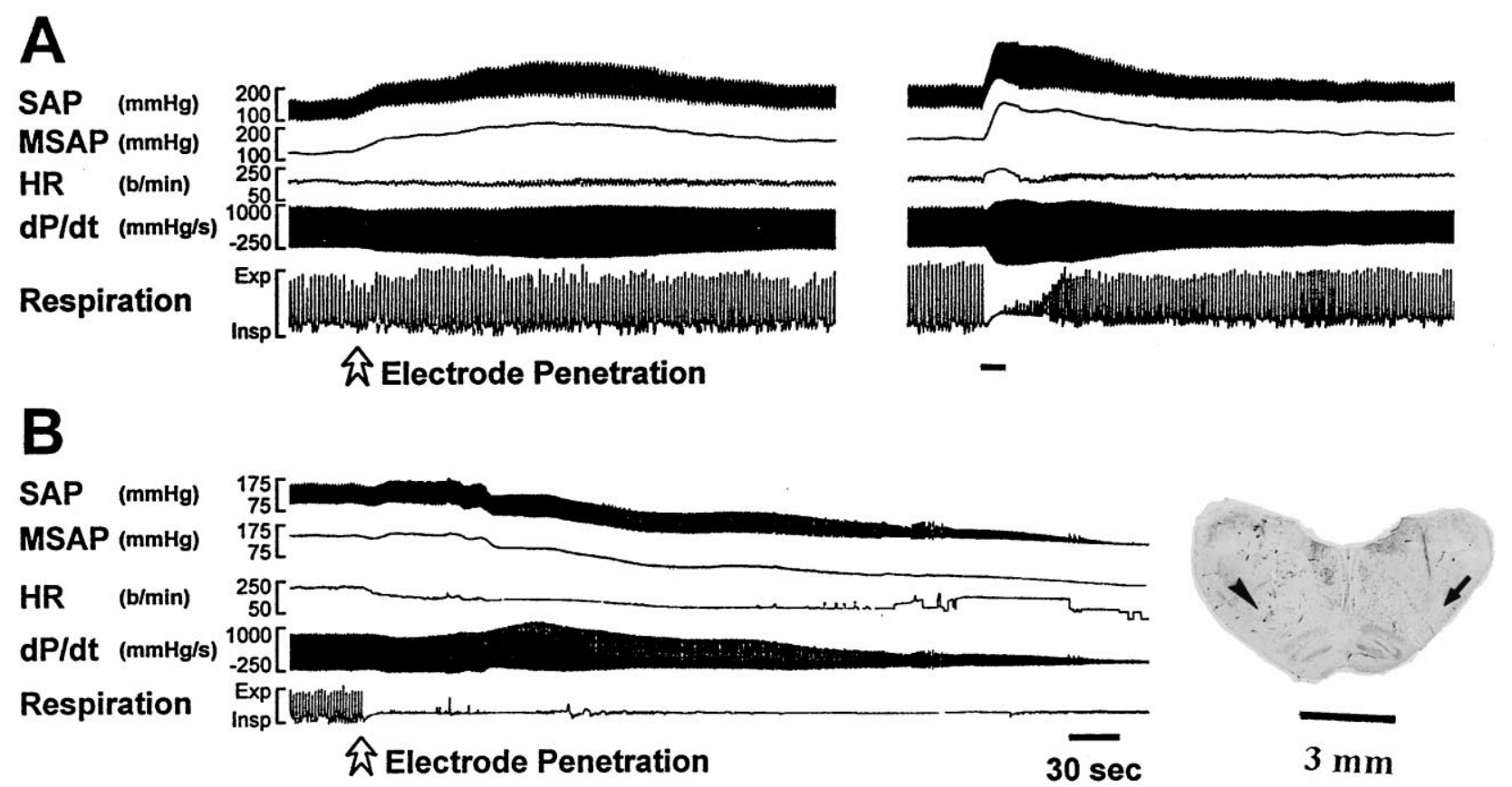

Fig. 6. Metal electrode-tubing penetration alone in VRG-APA produced persistent apnea under spontaneous breathing. (A) In a cat (970215) penetration in the ventrolateral medulla, lateral to the VRG-Apa with an electrode-tubing produced moderate rise in SAP (left panels) while electrical stimulation (80 $\mu \mathrm{A})$ to the same point produced a even higher but shorter increase of SAP associated with a brief inhibition of respiration (right panels). (B) In the same animal, inserting another electrode penetration into the opposite VRG-Apa alone without injection produced an immediate persistent apnea and death of the animal. Note the concomitant progressive falling of SAP and absence of marked gasping movement (respiration tracing) during the course of apnea, and persistent falling of SAP. 
5B). Microinjection of KA into the same VRG-Apa eventually produced complete apnea and death of the animal (Fig. 5C).

\subsection{Mechanical stimulation of the VRG-Apa}

\subsubsection{Metal electrode-tubing}

In 4 cats a previous electrode insertion was made in one side of the ventrolateral medulla that produced no apparent effects even after Glu microinjection. Histological examination later revealed that the location was not exactly on the VRG-Apa. These animals were subjected to a second electrode insertion into the contralateral VRG-Apa for chemical injection. In an average of $39 \pm 1 \mathrm{~s}$, the electrode insertion alone produced serious respiratory-cardiovascular dysfunction and eventually death of the animal (Fig. 4C, Fig. 6B).

In another 2 animals after 2-3 electrode penetrations in the ventralateral medulla during the process of localizing the VRG-Apa, single electrode penetration to contralateral medulla in VRG-Apa produced apnea and death of the animal.

\subsubsection{Glass micropipette}

Glass micropipette, $40-50 \mu$ in outside diameter, was used to stimulate the VRG-Apa in anesthetized and paralyzed animals under artificial ventilation for PNA recording. The procedure of micropipette insertion produced inhibition of the PNA only in one of the 5 animals, but PNA returned to the control level after 3 min (Fig. 2A). Gallamine was then discontinued in these animals and subsequent microinjection of KA (150 nl) in VRG-Apa produced persistent silence of PNA.

\subsection{Midsagittal division of the medulla}

Division at the rostral level: in 7 cats the medulla was longitudinally divided into two halves from the midline at an extent $0-5 \mathrm{~mm}$ rostral to the obex. After the division, the resting SAP was not altered, HR decreased but recovered within $2 \mathrm{~h}$. However, both PNAs were greatly inhibited and varied from an average reduction of $67 \%$ to complete inactivation (Fig. 7A). This inhibition persisted despite continuation of artificial ventilation for 3-4 h.

\section{YC970714}

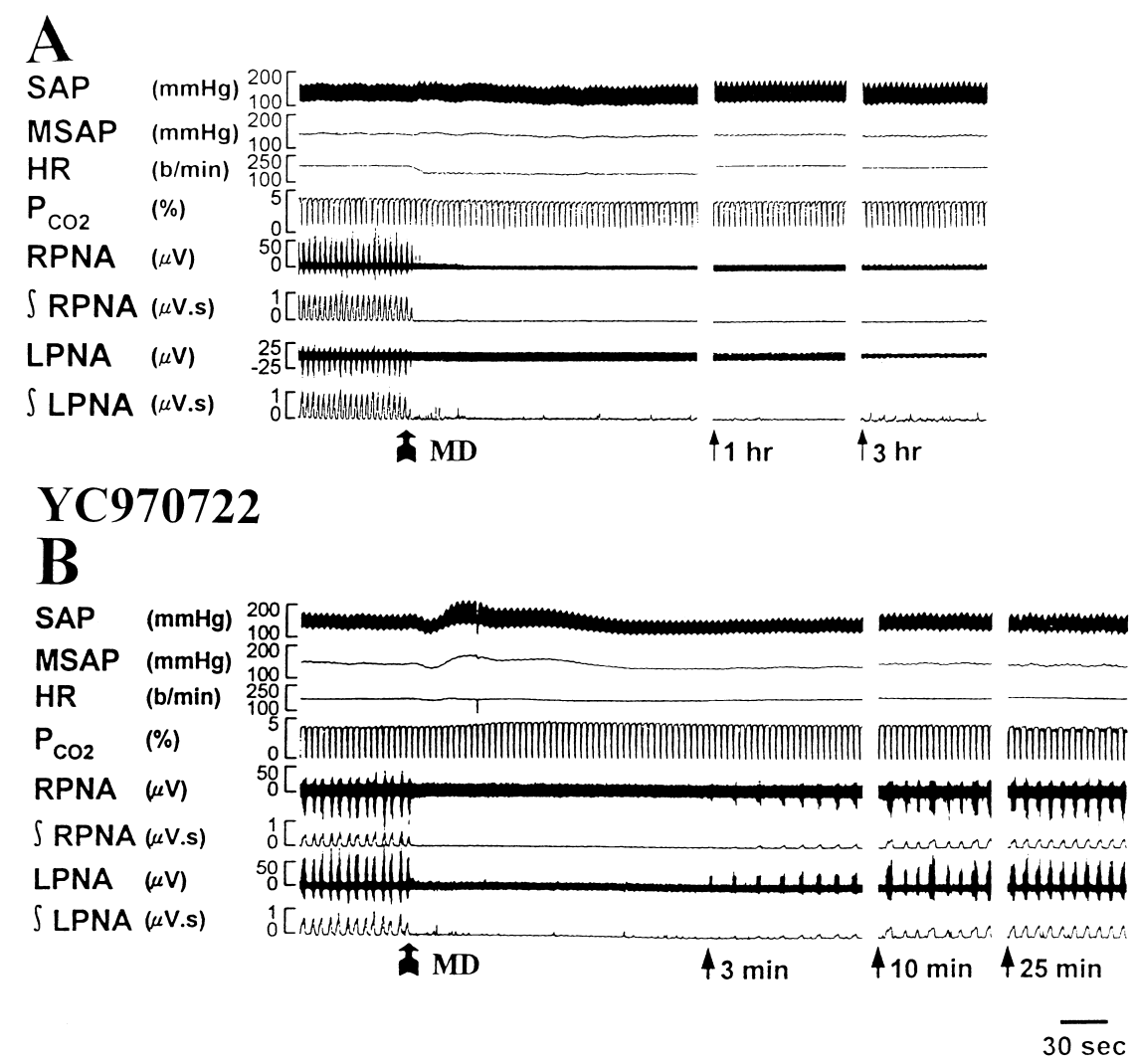

Fig. 7. Effects of midsagittal division of the medulla rostral or caudal to the obex on bilateral PNA. Upper panels from cat (CL970714): Upper panels show that midsagittal division in medulla $0-5 \mathrm{~mm}$ rostral to the obex produced persistent cessation of both PNA over $3 \mathrm{~h}$. Bottom panels from another cat (YC970722), in which midsagittal division was made 0-5 mm caudal to the obex. This produced only brief cessation of both PNAs but the latter returned 3 min later. 
Division at the caudal level: in another 4 cats midsagittal division of the medulla was made $0-5 \mathrm{~mm}$ caudal to the obex. The resting cardiovascular parameters were only transiently perturbed. PNA was also transiently abolished and recovered 3 min later (Fig. 7B).

\section{Discussion}

The VPR-Apa is important for maintaining normal respiration. In anesthetized but spontaneous breathing cats, microinjection of a small dose of Glu (100 nl) unilaterally into this area produced transient apnea, a larger dose of Glu (200 nl) produced slowing and irregular breathing, while a larger dose of KA (200 nl) often produced prolonged apnea. If PNA were recorded under artificial ventilation, such single-unilateral microinjection produced complete cessation of PNA on both sides, and the activity did not return despite the continuation of artificial ventilation for 3-4 h. Not only that, mere metal electrode penetration through VRG-Apa may sometimes cause respiratory failure. In all above conditions, stimulation of the VRG-Apa produced less marked cardiovascular changes. Therefore, the principal cause of death of such animals lies on respiratory rather than circulatory failure.

\subsection{Mechanical-minute lesion in VRG-Apa may cause apnea}

Electrode penetration into the brain inevitably causes mechanical irritation and/or damage of neural structures. The damage is more apparent and extensive when a larger coaxial electrode (Hytemco Precision) is used as in our earlier studies (Chai and Wang, 1962). Insertion of such a large electrode into the region of ventrolateral medulla near VRG-Apa often produces respiratory distress and occasionally fatal apnea (unpublished data). Using finer electrode-tubing in our recent studies decreased the frequency but not entirely eliminated the occurrence of such complication. If the electrode was not placed correctly but close to the VRG-Apa, then a second electrode insertion to the opposite VRG-Apa, produced persistent apnea. On the other hand, we never observed fatal cardiovascular collapse consequent to electrode penetration into the pressor areas in the dorsal or ventrolateral medulla. This suggests that the size of VRG-Apa responsible for the maintenance of normal-rhythmic respiration is much smaller, and/or its nature is more delicate, than that of the pressor areas for cardiovascular integration.

Similar respiratory depression has been reported by other investigators. Budzinska et al. (1985) reported that in cats insertion of a needle thermode for focal cooling the ventral medulla around the nucleus paragigantocellularis lateralis produced depression in respiration or apnea. Adams et al. (1988) reported that in dogs, insertion of a cryoprobe needle into the ventral and dorsal respiratory group for cooling the medulla often produced respiratory distress.

\subsection{The VRG-Apa is important for respiration}

The stimulation points in the present study fell in the rostral VRG including the pre-Bötzinger complex. The VRG is important for generation of respiratory rhythm (Bianchi et al., 1995; Paton et al., 1994). The VRG-Apa we studied includes the rostral part of VRG and the pre-Bötzinger complex, a transitional zone between the Bötzinger and the rostral VRG which modulate expiration and inspiration, respectively. The rostral VRG contains pre-inspiratory neurons, a most essential component for the genesis of respiratory rhythm (Connelly et al., 1992). The importance of the pre-Bötzinger complex for respiratory rhythm genesis has been shown by the following evidence. (1) In the in vitro brainstem preparations from the neonatal rat, which had been deprived from afferent inputs and synaptic connections, the respiratory rhythm was retained. In addition, in the tissue slice from the medulla containing the pre-Bötzinger complex, respiration related oscillation could be generated, in forms similar to those observed in the whole brainstem in vitro (Smith and Feldman, 1987; Smith et al., 1991). (2) The respiratory rhythm in the in vitro newborn rat preparation was preserved after removal of the GABAa or glycine receptor-mediated inhibitory synaptic transmission (Feldman and Smith, 1989; Smith and Feldman, 1987; Smith et al., 1991). Furthermore, in the neonatal brainstem-spinal cord preparation, after surgical isolation or blockade of the chemical synaptic transmission by bathing in low calcium and high magnesium medium, respiratory-like activity was observed in the preinspiratory neuron of the intermediorespiratory cells group (Onimaru et al., 1988, 1989). Brainstem cell cultures taken from the obex level of neonatal rats show periodic and aperiodic bioelectrical rhythm (Bingmann et al., 1991). Cultured-dissociated fetal rat cells from the nucleus ambiguous, $2 \mathrm{~mm}$ rostral to the obex, which are close to the ventral respiratory group, also show pacemaker activity with regular single or bursting activity (Rigatto et al., 1992).

\subsection{A single chemical lesion may be sufficient in causing persistent apnea}

Speck (1989), who microinjected KA $(0.1 \%)$ into both regions of the Bötzinger complex in cats, observed no alternation in the spontaneous activity of the left phrenic nerve. Besides, the inhibition of the left PNA consequent to stimulation of the central end of the right phrenic nerve with both threshold $(80 \mu \mathrm{A})$ and supra maximal $(15 \mathrm{~mA})$ intensity (phrenic-to-phrenic inhibitory response) was essentially unaltered. However, the phrenic inhibitory response was abolished after placing electrolytic lesion (DC, $20 \mu \mathrm{A}, 2 \mathrm{~min}$ ) on both sides of the Bötzinger complex. 
In the present study, microinjection of KA at a higher concentration $(0.51 \%)$ into unilateral VRG-Apa produced persistent apnea or silence of the PNA on both sides concomitant with an initial slight to moderate increase of SAP in 2/3 of the stimulations (see Figs. 1-3 and 5). This SAP increase appeared to be a result of sympathetic activation from the nearby pressor region (Chai et al., 1988; Wang et al., 1996). Even microinjecting a single large dose of Glu $(0.25 \mathrm{mM}, 200 \mathrm{nl})$ into the VRG-Apa area often caused strong respiratory depression that required artificial ventilation to rescue the animal. Similarly, Bongianni et al. (1993) microinjected DL-homocystic acid (160 mM, 10-30 nl) into unilateral ventrolateral medulla at the level extending from 2.4 to $6.3 \mathrm{~mm}$ rostral to the obex, covering the area of facial nucleus and retrofacial nucleus, causing inhibition of both PNAs over a period of $30 \mathrm{~min}$.

\subsection{Bilateral connection of the VRG}

Neurons in the VRG are reciprocally connected by fibers transversing the midline (Paton et al., 1994) and VRG-Apa on both sides are functioning as one integrated entity. Indeed, Onimaru et al. (1988) have shown, that in the brainstem-spinal cord preparation of newborn rats, synchronous burst activity between the right and left preinspiratory neurons must be above a certain level for triggering the inspiratory activity at $\mathrm{C} 4$. Thus, it is logical to consider that although each VRG can effect independent function, a certain extent of influence from both sides cannot be excluded, and dysfunction of one side will affect the function of the other. Histologically, after injection of the anterograde Fluoro Ruby into an unilateral rostral VRG in rats, various labelled fibers were found bilaterally in regions of the entire ventrolateral medulla related to respiration, i.e. the ambiguus, preambiguous, the lateral portion of the paragigantocellular nuclei, dorsal portion of the lateral reticular nucleus etc. (Gaytán et al., 1997). In other words, each nucleus responsible for respiration is closely connected with those located on the opposite side. In addition, fiber tracts from neurons responsible for respiration also cross the midline to the opposite side, i.e. the expiratory bulbospinal neurons in the Bötzinger complex; the inspiratory neurons of the dorsal respiratory group, the inspiratory neurons of the rostral ventral respiratory group and the expiratory bulbospinal neurons of the caudal respiratory group (Monteau and Hilare, 1991). Therefore, existence of heavy descending fiber tracts from both sides of the VRG (Bianchi et al., 1995) is a characteristic feature of respiratory integration, and is different from that of cardiovascular integration, in which fibers from cardiovascular neurons in the medulla descend predominantly ipsilaterally (Amendt et al., 1979). As a result, in spontaneously breathing animals, surgical division of the medulla along midline interrupts these crossing fibers and immediately produces irreversible apnea (Paton et al., 1994; Bianchi et al., 1995;
Guyenet and Koshiya, 1995), or in artificially ventilated animals cessation of bilateral PNAs. In contrast, sagittal division of the medulla does not affect the resting SAP and/or pressor responses subsequent to Glu activation of both dorsal medulla and rostral ventrolateral medulla $(\mathrm{Su}$ et al., 1992).

In the present study, midsagittal division was placed from $0-5 \mathrm{~mm}$ rostral to the obex. This resulted in persistent apnea and spontaneous breathing did not return even after a period of 3-4 h. This is consistent with the observations of Kubin et al. (1985) and Gromysz and Karczewski (1982) in artificially ventilated and paralyzed cats. Gromysz and Karczewslci divided the medulla from the midline, 2-14 $\mathrm{mm}$ rostral to the obex, and observed abolition of the PNA (Gromysz and Karczewski, 1982). Kubin et al. (1985) observed that midsagittal division in the medulla, extending from $3 \mathrm{~mm}$ caudal to $3 \mathrm{~mm}$ rostral to the obex, consistently decreased the activity of both phrenic and recurrent laryngeal nerves associated with or without change of the SAP and HR. If the division was rostrally extended 3-4 $\mathrm{mm}$ more, i.e. 6-7 $\mathrm{mm}$ from the obex, PNA was abolished, and only $5 \%$ of the recurrent laryngeal nerve activity remained. This may suggest that, in cats, fiber tracts responsible for respiration cross from both sides, and the linkage between neurons subserving generation of respiratory rhythm are heavily localized at the level 3-6 $\mathrm{mm}$ rostral to the obex. We observed that when midsagittal section was made at the level caudal to the obex, much less respiratory distress was produced suggesting a less condensed fiber pathways crossing at this level. However, in rats, Howard and Tabatabai (1975) reported that midsagittal section, $1 \mathrm{~mm}$ long at the level $0.5 \mathrm{~mm}$ caudal to the obex, decreased the respiratory rate from $77 / \mathrm{min}$ to $27 / \mathrm{min}$ with irregular rhythm and decrease of tidal volume to $40 \%$ of the control.

\subsection{VRG-Apa lesion may interfere with afferent activation}

It is noteworthy that no signs of struggling occurred during the period of VRG-Apa induced apnea in the anesthetized cats under spontaneous breathing or under artificial ventilation. Immediately after the onset of respiratory distress consequent to VRG lesion, the SAP increased slightly followed by a progressive fall to zero. During the whole period of apnea, animals did not show signs of struggling with increased muscle tone of limbs and violent or frequent gasping. The same occurred in the VRG-ApaApnea animals rescued by artificial ventilation but failed to start spontaneous breathing after removal of the respirator. This is quite different from anesthetized intact animals in apnea without VRG-Apa lesion. Signs of struggling may be seen in these animals. The VRG-Apa is located in the core of medullary reticular formation which was classically considered to be an ascending activation system (Magoun, 1963). It is not impossible that KA lesion in VRG-Apa blocks this system. 


\subsection{Microinjection of kynurenic acid (KYN) into the VRG-} Apa does not affect respiration

The genesis of the respiratory rhythm in the medulla involves endogenous release of excitatory amino acids. The latter acts principally on $N$-methyl-D-aspartate (NMDA) receptors (Richter et al., 1992). Both NMDA and non-NMDA receptors have been found in the phrenic neurons (Chitravanshi and Sapru, 1997). In the present study, KYN, a broad spectrum excitatory amino acid antagonist, was microinjected into the VRG-Apa with an attempt to examine whether blockade of its transmission will affect the respiration. Findings of the present study show that KYN, in a concentration of $0.1 \mathrm{M}$, did not affect the amplitude of respiration in most of the spontaneously breathing animals. In 4 animals, KYN, however, restored the decrease of SAP resulting from electrode-tubing insertion into the VRG-Apa (see Figs. 4 and 5). This may suggest that VRG-Apa contains some vasomotor-inhibitory neurons with amino acid receptors. KYN (175 pmol) microinjected bilaterally into the pre-Bötzinger area in rats abolishes the PNA and also the chemoreceptor reflex consequent to intravenous cyanide (Guyenet and Koshiya, 1995). The present study neither tested the effect of KYN on PNA nor on chemoreflex.

In summary, in a restricted region of VRG-Apa there exists a group of neurons essential for normal respiration. A single-minute damage on one side of this region resulting from electrode penetration may cause long lasting apnea, and administration of KA often result in death of the animal from persistent apnea. Normal respiration thus requires the integrity of neurons in VRG-Apa on both side of the medulla.

\section{Acknowledgements}

The authors express their gratitude to Drs K.K. Wu, T.K. Tang and T.L. Su for their encouragement and support during this study. We thank Mr G.T. Chen for preparation of illustrations and Ms J.J. Pan for preparation of the manuscript. Thanks are due to Drs S.H. Ngai, Columbia University, New York, and C.V. Weaver of Fu Jen Catholic University, Taipei, for comments of this paper. This study was support in part by the Foundation of Biomedical Sciences, Shih-Chun Wang Research Fund and the National Science Council, R.O.C., No. NSC87-2314-B-001002 .

\section{References}

Adams, M., Chonan, T., Cherniack, N.S., von Euler, C., 1988. Effects on respiratory pattern of focal cooling in the medulla of the dog. J. Appl. Physiol. 65, 2004-2010.

Amendt, K., Czachurski, J., Dembowsky, K., Seller, H., 1979. Bul- bospinal projections to the intermediolateral cell column; a neuroanatomical study. J. Auton. Nerv. Syst. 1, 103-117.

Bianchi, A.L., Denavit-Saubie, M., Champagnat, J., 1995. Central control of breathing in mammals: neuronal circuitry, membrane properties, and neurotransmitters. Physiol. Rev. 75, 1-45.

Bingmann, D., Baker, R.E., Ballantyne, D., 1991. Rhythm generation in brainstem cultures grown in a serum-free medium. Neurosci. Lett. 132, 167-170.

Bongianni, F., Corda, M., Fontana, G.A., Pantaleo, T., 1993. Excitatory and depressant respiratory responses to chemical stimulation of the rostral ventrolateral medulla in the cat. Acta Physiol. Scand. 148, 315-325.

Budzinska, K., von Euler, C., Kao, F.F., 1985. Effects of graded focal cold block in rostral areas of the medulla. Acta. Physiol. Scand. 124, 329-340.

Chai, C.Y., Lin, R.H., Lin, A.M.Y., Pan, C.M., Lee, E.H.Y., Kuo, J.S., 1988. Pressor responses from electrical or glutamate stimulations of the dorsal or ventrolateral medulla. Am. J. Physiol. 255, R709-R717.

Chai, C.Y., Mu, J.Y., Brobeck, J.R., 1965. Cardiovascular and respiratory responses from local heating of medulla oblongata. Am. J. Physiol. 209, 301-306.

Chai, C.Y., Wang, S.C., 1962. Localization of central cardiovascular control mechanism in lower brain stem of the cat. Am. J. Physiol. 202, 25-30.

Chitravanshi, V.C., Sapru, H.N., 1997. NMDA as well as non-NMDA receptors in phrenic nucleus mediate respiratory effects of carotid chemoreflex. Am. J. Physiol. 272, R302-R310.

Connelly, C.A., Dobbins, E.G., Feldman, J.L., 1992. Pre-Bötzinger complex in cats: respiratory neuronal discharge patterns. Brain Res. 590, 337-340.

Feldman, J.L., Smith, J.C., 1989. Cellular mechanism underlying modulation of breathing pattern in mammals. In: Davis, M., Jacobs, B.L., Schpenfeld, R.I. (Eds.), Modulation of Defined Vertebral Neural Circuit. Ann. N.Y. Acad. Sci. 563, 114-130.

Gaytán, S.P., Pedro, F.C., Núñez-Abades, A., Morillo, A.M., Pásaro, R., 1997. Pontomedullary efferent projections of the ventral respiratory neuronal subsets of the rat. Brain Res. Bull. 42, 323-334.

Gromysz, H., Karczewski, W.A., 1982. Phrenic motoneurone activity in split-brainstem cats and monkeys. Respir. Physiol. 50, 51-61.

Guyenet, P.G., Koshiya, N., 1995. Working model of the sympathetic chemoreflex in rats. Clin. Exp. Hypertens. 17, 167-179.

Howard, B.R., Tabatabai, M., 1975. Localization of the medullary respiratory neurons in rats by microelectrode recording. J. Appl. Physiol. $39,812-817$.

Hwang, J.J., 1994. Correlation between the pressor areas of the periaqueductal grey and the cardiovascular reactive areas of medulla in cats. Master of Science thesis of the National Yang-Ming Medical College (Supervisor C.Y. Chai).

Hwang, J.C., Su, C.K., Yen, C.T., Chai, C.Y., 1992. Presence of neuronal cell bodies in the sympathetic pressor areas of dorsal and ventrolateral medulla inhibiting phrenic nerve discharges in cats. Clin. Auton. Res. 2, 189-196.

Kubin, L., Trzebski, A., Lipski, J., 1985. Split medulla preparation in the cat: arterial chemoreceptor reflex and respiratory modulation of the renal sympathetic nerve activity. J. Auton. Nerv. Syst. 12, 211-225.

Magoun, H.W., 1963. The Waking Brain, 2nd edn. Charles C. Thomas, Spring Field.

Monteau, R., Hilare, G., 1991. Spinal respiratory motoneurons. Prog. Neurobiol. 37, 83-144.

Onimaru, H., 1995. A studies of the respiratory center using isolated brainstem-spinal cord preparation. Neurosci. Res. 21, 183-190.

Onimaru, H., Arata, A., Homma, I., 1988. Primary respiratory rhythm generator in the medulla of brainstem spinal cord preparations from newborn rats. Brain Res. 445, 314-324.

Onimaru, H., Arata, A., Homma, I., 1989. Firing properties of respiratory rhythm generating neurons in the absence of synaptic transmission in rat medulla in vitro. Exp. Brain Res. 76, 530-536. 
Paton, J.F.R., Ramirez, J.-M., Richter, W., 1994. Mechanism of respiratory rhythm generation change profoundly during early life in mice and rats. Neurosci. Lett. 170, 167-170.

Richter, D.W., Ballangi, K., Schwarzacher, S., 1992. Mechanism of respiratory rhythm generation. Curr. Opin. Neurobiol. 2, 788-793.

Rigatto, H., Fitzgerald, S.C., Willis, M.A., Yu, C., 1992. In search of the central respiratory neurons: II. Electrophysiologic studies of medullary fetal cells inherently sensitive to $\mathrm{CO}_{2}$ and low pH. J. Neurosci. Res. 33, 590-597.

Smith, J.C., Feldman, J.L., 1987. Central respiratory pattern generation studied in an in vitro mammalian brainstem spinal cord preparation. In: Sieck, G.C., Gandevia, S.G., Cameron, W.E. (Eds.), Respiratory Muscles and Their Neuromotor Control. Liss, New York, pp. 27-36. Smith, J.C., Ellenberger, H.H., Ballanyi, K., Richter, D.W., Feldman,
J.L., 1991. Pre-Bötzinger complex: a brainstem region that may generate respiratory rhythm in mammals. Science $254,726-729$.

Speck, D.F., 1989. Bötzinger complex region role in phrenic-to-phrenic inhibitory reflex of cat. J. Appl. Physiol. 67, 1364-1370.

Su, C.K., Chen, S.Y., Yang, J.M., Lin, R.H., Horng, H.T., Hwang, J.C., Chai, C.Y., 1992. Correlation of cardiovascular and respiratory responses to glutamate excitation of pressor areas of the medulla in cats. Chin. J. Physiol. 35, 279-289.

Wang, S.D., Yen, K.D., Pan, C.M., Liu, J.C., Kuo, J.S., Chai, C.Y., 1996. Descending pressor pathways from the dorsomedial and ventrolateral medulla of cats. Chin. J. Physiol. 39, 31-42.

Wang, S.C., 1980. Physiology and Pharmacology of the Brain Stem. Futura Publishing, New York. 